\title{
SCALE-INVARIANT CORRELATION FUNCTIONS OF COSMOLOGICAL DENSITY FLUCTUATIONS IN THE STRONG CLUSTERING REGIME AND THE STABILITY
}

\author{
TAIHEI YANO AND NAOTERU GOUDA \\ Department of Earth and Space Science, Graduate School of \\ Science, Osaka University, Toyonaka 560, Japan
}

We have investigated the scale-invariant solutions of the BBGKY equations for spatial correlation functions of cosmological density fluctuations and the mean relative peculiar velocity in the strongly nonlinear regime. It is found that the solutions for the mean relative physical velocity depend on the three-point spatial correlation function and the skewness of the velocity fields. We find that the stable condition in which the mean relative physical velocity vanishes on the virialized regions is satisfied only under the assumptions which Davis \& Peebles took in there paper. It is found, however, that their assumptions may not be general in real. The power index of the two-point correlation function in the strongly nonlinear regime depends on the mean relative peculiar velocity, the three-point correlation function and the skewness. If self-similar solutions exist, then the power index in the strongly nonlinear regime is related to the power index of the initial power spectrum and its relation depends on the three-point correlation function and the skewness through the mean relative peculiar velocity. We also investigate stability of the solutions of the BBGKY equations for two-point spatial correlation functions. In the case that the background skewness is equal to 0 , we found that there is no local instability in the strongly non-linear regime.

\section{References}

M.Davis and P.J.E.Peebles, ApJS, 34, 425 (1977)

T.Yano and N.Gouda, ApJ, 487, 473 (1997)

T.Yano and N.Gouda, ApJ, 495, 533 (1998) 